\title{
Adaptive Tracking Control of a Virtual Player in the Mirror Game
}

\author{
Chao Zhai ${ }^{1}$, Francesco Alderisio ${ }^{1}$, Krasimira Tsaneva-Atanasova ${ }^{2}$ and Mario di Bernardo ${ }^{1}$
}

\begin{abstract}
The coordination of interpersonal rhythmic movements is of great significance due to its potential relevance to human motor rehabilitation. In this paper we consider the problem of designing a controller able to drive a virtual player (VP) capable of imitating and following a human player in the mirror game [1]. The classic nonlinear HakenKelso-Bunz (HKB) model is adopted to describe the social motor coordination between two players. An adaptive control algorithm is developed and implemented on the HKB model to drive the VP. It is proven that the position error between the VP driven by our control algorithm and the human player is upper bounded during the game. Finally, experiments are conducted on a prototype set-up in order to evaluate the performance of the proposed control algorithm and compare it with other existing algorithms.
\end{abstract}

\section{INTRODUCTION}

The mirror game has been proposed as a simple, yet powerful paradigm for studying social motor coordination between two human players, e.g. [1] and references therein. In its simplest formulation, the mirror game features two people imitating each other's movements at high temporal and spatial resolution. It can be played in two different conditions: Leader-Follower, where one of the players (the follower) attempts at tracking the motion of the other player (the leader) as accurately as possible, and Joint Improvisation, involving the players jointly coordinating and synchronizing their movements without any of the two being designated as leader or follower.

The mirror game is a paradigm of human coordination in many areas of human endeavour as for example improvisation theater, sport, or dance. It is also particularly relevant in motor rehabilitation where patients are required to replicate movements shown to them by a physiotherapist [2].

Multiple studies in social psychology reveal that people prefer to interact with others who are similar to themselves morphologically and behaviorally [3]. Moreover, much evidence shows there exists a link between motor processes in interpersonal coordination and intra-personal mental connectedness [4]. People tend to unconsciously coordinate their movement and match their postures during interaction, enhancing interpersonal affinity [5]. For example, activities involving motor coordination and synchrony with others

\footnotetext{
${ }^{1}$ Chao Zhai, Francesco Alderisio and Mario di Bernardo are with Department of Engineering Mathematics, University of Bristol, Queen's Building, BS8 1TR, United Kingdom. chao. zhai@bristol.ac.uk, f.alderisiodbristol.ac.uk Mario di Bernardo is also with Department of Electrical Engineering and Information Technology, University of Naples Federico II, 80125 Naples, Italy. m.dibernardo@bristol.ac.uk

${ }^{2}$ Krasimira Tsaneva-Atanasova is with College of Engineering, Mathematics and Physical Sciences, University of Exeter, United Kingdom. $\mathrm{k}$.tsaneva-atanasova@exeter.ac.uk
}

(such as marching, singing and dancing) foster social attachment and thus increase cooperation among group members [6].

For this reason, it has been suggested that, rather than having two people performing the game, it might be desirable for the mirror game to be played by a virtual player, or avatar, and a human being (typically the patient). In this way, it is possible to control the features of the VP movement and posture and hence its similarity. It is also promising to use the interaction with the VP to better understand human coordination and social interaction as suggested in [7], [8] where the notion of human dynamic clamp (HDC) is introduced.

The mirror game with a VP can be formulated as a control problem where the aim is to design a control law able to drive the VP so that the desired game condition is achieved. Much research has been carried out in this direction as for example within the scope of the European project "AlterEgo" [9]. The grand open challenge is to design a controller able to decide the motion of the VP so as to make it capable of tracking or leading the human patient while exhibiting kinematic properties similar to those observed in the movement of human beings.

Motor coordination between multiple end effectors in biological systems has been widely investigated in the past decades [10], [11], [12], [13]. Such coordination emerges at many levels of the motor control hierarchy [14], and it can be achieved through feed-forward and feedback control in motor systems [15]. Feed-forward control serves to reconcile the interdependence of effectors in a preplanned way so that behavioral goals can be achieved successfully. In addition, motor systems are able to react to the sensory input and rectify the deviation from intended movements with the aid of feedback control.

In this paper we investigate the mirror game played in a Leader-Follower condition. Specifically, the human player acts as a leader while the avatar acts as a follower. As a consequence, our goal is to design a control law for the VP to track the leader's trajectory while showing human-like behavior. To solve this problem, an adaptive nonlinear control strategy is designed to update the coupling parameters in a nonlinear HKB oscillator as a function of the measured human position and estimated velocity. It is shown that the output of this equation can effectively track the human player and hence provide trajectories for the end effector of a robot or virtual avatar playing the game with a human subject.

A simple, yet effective experimental set-up, is developed and used to test and validate the performance of the control algorithm. A comparison with the performance of the 
reactive-predictive controller presented in [1] is also included to further emphasize the effectiveness of the control strategy.

\section{HKB MODEL}

In order to describe the motor coordination between a human player and a virtual player while performing the mirror game, it is necessary to model the dynamics of the former as accurately as possible.

Several papers are available in the literature that study human motor coordination. Much research attention has been focused on human bimanual coordination [11], [16], [17]. It has been found that when a human player is asked to oscillate his/her index fingers (or drive some manipulandum) synchronously, several nonlinear phenomena occur as the player is instructed to increase the cycling frequency. In particular, letting $\phi=\phi_{1}-\phi_{2}$ be the relative phase between the oscillations of the two fingers, only two stable modes were detected associated to $\phi=0$ (symmetric mode) and $\phi=\pi$ (anti-symmetric mode). Also, an abrupt hysteretic phase transition from one mode to the other is tipically observed above a critical value of the frequency [11].

The Haken-Kelso-Bunz (HKB) model was proposed in [18] as being able to capture all of the observed experimental features and also describe coordination between two players [7], [8]. The model consists of two nonlinearly coupled oscillators described by

$$
\ddot{z}+\left(\alpha \dot{z}^{2}+\beta z^{2}-\gamma\right) \dot{z}+\omega^{2} z=\left[a+b(z-w)^{2}\right](\dot{z}-\dot{w})
$$

where $z, \dot{z}$ represent the position and velocity of the end effector of player $1, w, \dot{w}$ the position and velocity of that of player 2 (modelled by a replica of the equation above obtained by swapping $w, \dot{w}$ with $z, \dot{z}) ; a$ and $b$ are the coupling parameters and $\alpha, \beta, \gamma$ and $\omega$ characterize the response of each uncoupled player when subject to some reference signal. In our paper we take (1) as the model describing the intrinsic dynamics $(z, \dot{z})$ of the VP coupled to that of the human player encoded in $(w, \dot{w})$

\section{PROBLEM STATEMENT}

Our set-up is inspired by the one in [1]. Specifically, we consider the motion of two identical masses (e.g. balls) sliding onto two parallel strings with the same length $l$ (see Fig. 11. In this implementation of the mirror game, two players (a human player and a virtual player) are required to move the ball back and forth along the string and synchronize their movement. Here, we assume that the game is played in a Leader-Follower condition, where the human player is the leader and the virtual player (robot or computer avatar) is the follower trying to track the leader movement.

The position of the ball moved by the human player is detected by a position sensor. A feedback control strategy then needs to be designed in order to generate the trajectory of the ball moved by the virtual player so as to track the movement of the ball controlled by the leader. Such a trajectory can then be provided to the on-board controllers of the VP (robot or computer avatar) as the desired trajectory for its end effector (see Fig. 2).

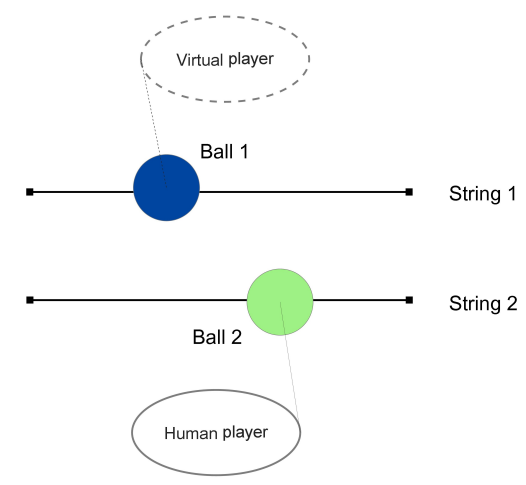

Fig. 1. Experimental set-up in the mirror game.

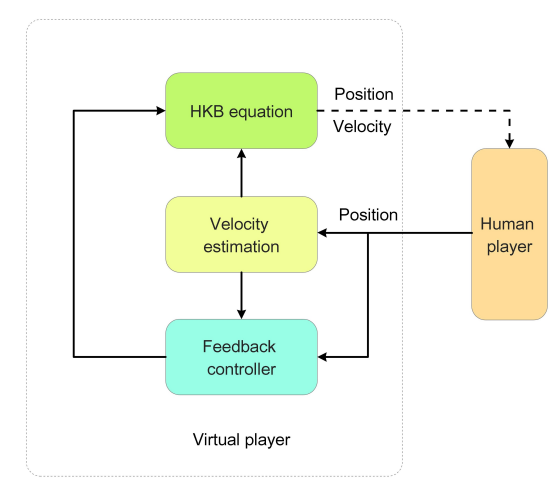

Fig. 2. Schematic diagram for the generation of the tracking trajectory of virtual player.

To make sure the movement of the virtual player presents features typical of human motor coordination in terms of reaction time, velocity distribution and so forth, we propose to generate the VP motion by controlling the dynamics of the nonlinear HKB model (1) to generate the position and velocity of its end-effector (driving the ball on the string).

The VP problem can then be formulated as the following control problem. Specifically, given a nonlinear HKB model of the form

$$
\left\{\begin{array}{l}
\dot{x}=y \\
\dot{y}=-\left(\alpha y^{2}+\beta x^{2}-\gamma\right) y-\omega^{2} x+u(x, y, w, \dot{w})
\end{array}\right.
$$

where $x, y$ and $w, \dot{w}$ represent position and velocity of the VP and the human player, respectively, and $u$ is an external control input modeling the coupling between the two players, the problem is then to design a feedback controller $u$ (coupling) such that the position $x(t)$ of the VP achieves bounded asymptotic tracking of the position of the human player $w(t)$, while preserving the features of human motor coordination described in the literature [1], [11], [17], [18], [19], [20], [21]. 


\section{CONTROL ARCHITECTURE}

To solve the problem stated above, we propose to use a strategy based on the model of the interaction between two human players in (11). Specifically, we choose the nonlinear controller given by

$$
u=\left[a(t)+b(t)\left(x-r_{p}\right)^{2}\right]\left(\dot{x}-\hat{r}_{v}\right)-C_{p} e^{-\delta\left(\dot{x}-\hat{r}_{v}\right)^{2}}\left(x-r_{p}\right)
$$

where $r_{p}$ is the position of the human player, $\hat{r}_{v}$ is the estimated velocity, $C_{p}$ and $\delta$ are constant positive parameters while the coupling parameters $a$ and $b$ are given by the adaptive laws:

$$
\dot{a}=-e^{-2 a}\left[\left(x-r_{p}\right)\left(y-\hat{r}_{v}\right)+\eta\left(x-r_{p}\right)^{2}\right]-\eta
$$

and

$$
\dot{b}=\frac{y-\hat{r}_{v}}{e^{2 b}}\left[\omega^{2} x+\left(\alpha y^{2}+\beta x^{2}-\gamma\right) y-\eta\left(y-\hat{r}_{v}\right)-u\right]-\eta
$$

where $\eta$ is a positive constant. Note that the control law (3) consists of two terms. The first term has the same structure as the coupling proposed in [18] to model interaction between two human players, albeit with the introduction of adaptive parameters to account for variability between different players. The second term deals with the position error when the velocity mismatch approaches zero and hence the first term decays to zero as well. When $\left|\dot{x}-\hat{r}_{v}\right|$ is relatively large, the first term in 3 becomes dominant and motor coordination between the two players becomes more pronounced during the mirror game.

Before presenting the validation of this approach, we present its theoretical stability analysis. We assume that the velocity of the human player is estimated by the following backward difference rule:

$$
\hat{r}_{v}(t) \triangleq \frac{r_{p}(k T)-r_{p}(k T-T)}{T}, \quad t \in[k T,(k+1) T)
$$

where $T$ is the sampling time period of the position sensor detecting the position of the ball moved by the human player, and $r_{p}(k T), k \in \mathbb{N}^{*}$ represents the sampling value of the ball position at time $k T$. Clearly, $\hat{r}_{v}(t)$ is piece-wise constant at each sampling period. Note that $\hat{r}_{v}(t) \rightarrow r_{v}(t)$ as $T \rightarrow 0$.

For the sake of simplicity, we adopt the following linear model to describe the motion of the ball moved by the human player:

$$
r_{p}(t)=r_{p}(k T)+\hat{r}_{v}(t)(t-k T), \quad t \in[k T,(k+1) T)
$$

and suppose that the sampling period $T$ is sufficiently small so that the error between the estimated position in the linear model and the actual position of the ball is negligible. Here, a zero-order hold velocity estimator is used to predict the movement of the human player. Note that the use of other more sophisticated rules for velocity estimation is also possible, such as the use of nonlinear observers [22].

Proposition 4.1: The adaptive feedback controller (3) with the linear model (4) ensures that the solution of the controlled HKB model (2) satisfies

$$
\left|x(t)-r_{p}(t)\right| \leq e^{\eta T} \sqrt{\frac{2 \varepsilon}{e^{2 \eta T}-2}}+\frac{2}{e^{\eta T}} \sqrt{H(0)}, \quad t \in[0,+\infty)
$$

if $\eta$ is chosen so that

$$
\eta>\frac{\ln 2}{2 T}
$$

where

$H(0)=\frac{1}{2}\left[\left(x(0)-r_{p}(0)\right)^{2}+\left(y(0)-\hat{r}_{v}(0)\right)^{2}+e^{2 a(0)}+e^{2 b(0)}\right]$

and

$$
\varepsilon=\sup _{k \in \mathbb{N}^{*}}\left(T^{2}+1\right)\left(\hat{r}_{v}(k T)-\hat{r}_{v}(k T-T)\right)^{2} .
$$

Proof: Choose the energy-like function

$$
H \triangleq \frac{1}{2}\left[\left(x-r_{p}\right)^{2}+\left(y-\hat{r}_{v}\right)^{2}+e^{2 a}+e^{2 b}\right]
$$

Note that $\hat{r}_{v}$ is fixed in each sampling interval $[k T,(k+$ 1)T), $k \in \mathbb{N}^{*}$. Then the time derivative of $H$ along the trajectories of (2) with $u$ defined in (3) is given by

$$
\begin{aligned}
\dot{H} & =\left(x-r_{p}\right)\left(\dot{x}-\hat{r}_{v}\right)+\left(y-\hat{r}_{v}\right) \dot{y}+e^{2 a} \dot{a}+e^{2 b} \dot{b} \\
& =\left(x-r_{p}\right)\left(y-\hat{r}_{v}\right)-\left(y-\hat{r}_{v}\right)\left[\left(\alpha y^{2}+\beta x^{2}-\gamma\right) y+\omega^{2} x-u\right] \\
& +\left(y-\hat{r}_{v}\right)\left[\omega^{2} x+\left(\alpha y^{2}+\beta x^{2}-\gamma\right) y-\eta\left(y-\hat{r}_{v}\right)-u\right]-\eta e^{2 b} \\
& -\left[\left(x-r_{p}\right)\left(y-\hat{r}_{v}\right)+\eta\left(x-r_{p}\right)^{2}\right]-\eta e^{2 a} \\
& =-\eta\left(x-r_{p}\right)^{2}-\eta\left(y-\hat{r}_{v}\right)^{2}-\eta e^{2 a}-\eta e^{2 b} \\
& =-2 \eta H, \quad t \in[k T,(k+1) T)
\end{aligned}
$$

Solving the above differential equation yields

$$
H(t)=e^{-2 \eta(t-k T)} H(k T), \quad t \in[k T,(k+1) T)
$$

Moreover, at the sampling point $k T$ we have

$$
\begin{aligned}
& H(k T)-H^{-}(k T) \\
= & \frac{1}{2}\left[\left(x-r_{p}(k T)\right)^{2}-\left(x-r_{p}(k T-T)-\hat{r}_{v}(k T-T) T\right)^{2}\right. \\
+ & \left.\left(y-\hat{r}_{v}(k T)\right)^{2}-\left(y-\hat{r}_{v}(k T-T)\right)^{2}\right] \\
\leq & \left(r_{p}(k T)-r_{p}(k T-T)-\hat{r}_{v}(k T-T) T\right)^{2} \\
+ & \left(\hat{r}_{v}(k T)-\hat{r}_{v}(k T-T)\right)^{2}+H^{-}(k T) \\
= & \left(1+T^{2}\right)\left(\hat{r}_{v}(k T)-\hat{r}_{v}(k T-T)\right)^{2}+H^{-}(k T)
\end{aligned}
$$

which is equivalent to

$$
H(k T) \leq \varepsilon+2 H^{-}(k T)
$$

Here

$$
\varepsilon=\sup _{k \in \mathbb{N}^{*}}\left(T^{2}+1\right)\left(\hat{r}_{v}(k T)-\hat{r}_{v}(k T-T)\right)^{2}
$$

and

$$
H^{-}(k T)=\lim _{t \backslash k T} H(t)
$$

Evaluating (6) and (7) at $t=(k+1) T$ and nesting the inequalities backwards till $t=0$, we get

$$
\begin{aligned}
H(k T) & \leq \varepsilon\left[1+\frac{2}{e^{2 \eta T}}+\left(\frac{2}{e^{2 \eta T}}\right)^{2}+\ldots+\left(\frac{2}{e^{2 \eta T}}\right)^{k-1}\right] \\
& +\left(\frac{2}{e^{2 \eta T}}\right)^{k} H(0) \\
& =\varepsilon \frac{1-\left(\frac{2}{e^{2 \eta T}}\right)^{k}}{1-\frac{2}{e^{2 \eta T}}}+\left(\frac{2}{e^{2 \eta T}}\right)^{k} H(0) \\
& \leq \frac{\varepsilon}{1-\frac{2}{e^{2 \eta T}}}+\frac{2}{e^{2 \eta T}} H(0), \quad \forall k \in \mathbb{N}^{*}
\end{aligned}
$$


when the inequality $\eta>\frac{\ln 2}{2 T}$ holds. Moreover, combining 6 with (8), we get

$$
H(t) \leq \frac{\varepsilon}{1-\frac{2}{e^{2 \eta T}}}+\frac{2}{e^{2 \eta T}} H(0), \quad t \in[0,+\infty)
$$

which clearly implies

$$
\left|x(t)-r_{p}(t)\right| \leq e^{\eta T} \sqrt{\frac{2 \varepsilon}{e^{2 \eta T}-2}}+\frac{2}{e^{\eta T}} \sqrt{H(0)}, \quad t \in[0,+\infty)
$$

Remark 4.1: It is easy to demonstrate that the coupling parameters $a$ and $b$ are upper bounded with the proposed adaptive laws.

Remark 4.2: Since $r_{p}(t) \in[0, l], \forall t \geq 0$ and $\left|\hat{r}_{v}(t)\right| \leq \frac{l}{T}$, the following inequality holds

$$
\varepsilon=\sup _{k \in \mathbb{N}^{*}}\left(T^{2}+1\right)\left(\hat{r}_{v}(k T)-\hat{r}_{v}(k T-T)\right)^{2} \leq \frac{4 l^{2}\left(1+T^{2}\right)}{T^{2}}
$$

Generally, the upper bound for the position error is relatively conservative. When the velocity of the human player is small, $\varepsilon$ is small as well, and the estimation for the position error is accurate enough. In addition, taking the limit of (8) as $k T \rightarrow \infty$ and combining it with (5), the position error between the two players satisfies the following inequality:

$$
\limsup _{k T \rightarrow+\infty}\left|x(t)-r_{p}(t)\right| \leq e^{\eta T} \sqrt{\frac{2 \varepsilon}{e^{2 \eta T}-2}}
$$

Similarly, we can estimate the velocity error as

$$
\begin{gathered}
\left|\dot{x}(t)-\hat{r}_{v}(t)\right| \leq e^{\eta T} \sqrt{\frac{2 \varepsilon}{e^{2 \eta T}-2}}+\frac{2}{e^{\eta T}} \sqrt{H(0)}, \quad t \in[0,+\infty) \\
\text { V. EXPERIMENTS }
\end{gathered}
$$

In this section we experimentally validate our approach on a simple, yet effective, set-up implemented at the University of Bristol. We also compare its performance with the reactive-predictive controller proposed in [1].

The employed set-up was developed for measuring motions of players in the one-dimensional mirror game. A human participant is required to lead the game while interacting with our VP (running on a laptop computer). In order to detect the position of the human player hand, a leap motion controller [23] is used (see Fig. 3).

The leap motion controller and the laptop computer are both placed on a table whose height is around $70 \mathrm{~cm}$. The human player is required to wave his/her hand horizontally over the leap motion controller at a vertical distance of approximately $50 \mathrm{~cm}$. Indeed, at this distance the horizontal resolution of the device is maximum and it is able to measure the hand position within a range of $60 \mathrm{~cm}$. The position of the hand of the human player within this interval is mapped into the interval $[-0.5,0.5]$ and visualized on the computer screen as a green solid circle, while the position of the virtual player is visualized as a blue solid circle. The control algorithm is implemented in MATLAB (version R2012b) [24].

Players can be either standing or seated. After the game is initialized, there is a $2 \mathrm{~s}$ wait before data recording begins and the game starts. This initial delay is used to allow the human

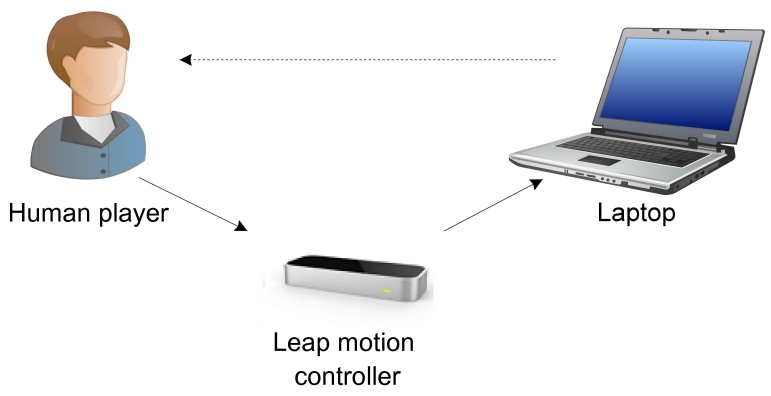

Fig. 3. Experimental equipment in the mirror game between human player and virtual player. The position of the human finger is detected by a leap motion controller and sent to the laptop, while the position of the virtual player is calculated by running the corresponding Matlab code. Two balls corresponding to the human player and the virtual player positions, respectively, are shown on the laptop screen.

player to place his/her hand over the leap motion controller. Human players are not instructed before playing the game, but they are just told to act as a leader and let the VP follow them during a 60s round.

Parameters for the HKB equation and the adaptive feedback controller (AFC) in (3) are set heuristically as follows: $\alpha=10, \beta=20, \gamma=-1, \omega=0.1, a(0)=-5, b(0)=-5$, $C_{p}=40$ and $\delta=0.25$. In our implementation the sampling time is $T=0.1 \mathrm{~s}$ and therefore $\eta=30>\frac{\ln 2}{2 T} \simeq 0.35$. In particular, the values of all the previous parameters have been chosen so that the response of the HKB oscillator to several sinusoidal signals with different frequencies is qualitatively the same as the one that was prerecorded for a human player trying to track the same references. Moreover, it is worth pointing out that the initial values of $a$ and $b$ influence the performance of the avatar only at start-up.

The reactive-predictive controller (RPC) proposed in [1] is also implemented to compare its performance against that of our adaptive feedback controller when considering the same input trajectory from the human leader. Following the scheme presented in [1] to implement the RPC, the dynamics of the $\mathrm{VP}$ is described by the following system:

$$
\ddot{x}=\sum_{i=1}^{5} A_{i} \omega_{i} \cos \left(\omega_{i} t\right)+f
$$

where $x \in \mathbb{R}$ represents the position of the avatar and

$$
\dot{f}=k\left(\hat{r}_{v}-\dot{x}\right), \quad k>0
$$

with the parameters $A_{i}$ being estimated adaptively as

$$
\dot{A}_{i}=\lambda\left[\hat{r}_{v}-\sum_{i=1}^{5} A_{i} \sin \left(\omega_{i} t\right)\right] \sin \left(\omega_{i} t\right), \quad \lambda>0
$$

As suggested in [1], in this case the parameters are chosen as follows: $\omega_{1}=0.025, \omega_{2}=0.05, \omega_{3}=0.075, \omega_{4}=0.1$, $\omega_{5}=0.125, \lambda=0.01, k=30$ and $A_{i}(0)=0, \forall i=1, \ldots, 5$.

As we can see from Figures 4 and 5 , the adaptive control strategy presented in this paper guarantees good tracking 


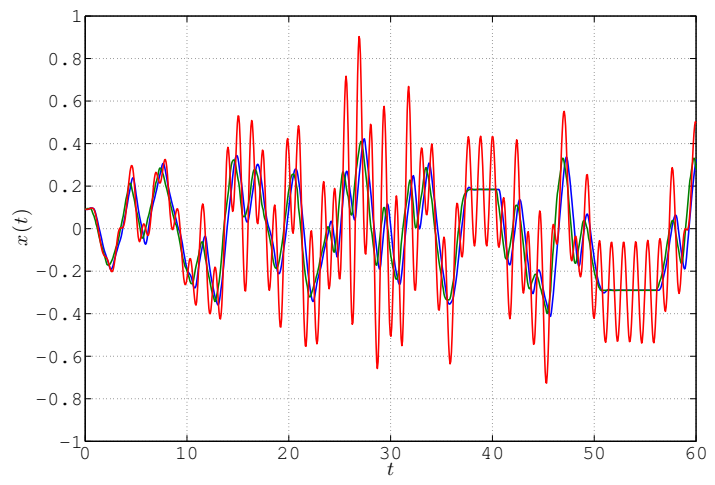

(a) position time series

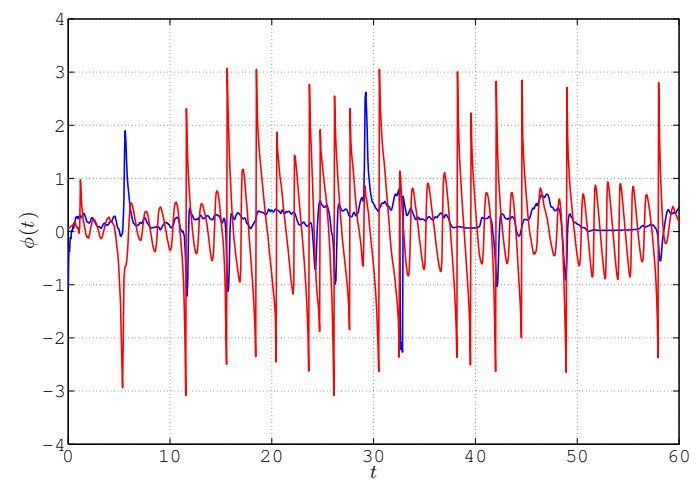

(b) relative phase time series

Fig. 4. Time series of the position (a) and of the relative phase (b) between the human leader and the VP; blue (AFC), red (RPC), green (human leader)

with a performance that is generally better than that of the RPC strategy presented in [1]. To compare the performance of the two algorithms, we plot the time series of both the position and the relative phase together with the distributions of velocitiy and relative phase of the human and the virtual player. In particular, the relative phase between the two players is defined as $\phi=\phi_{H L}-\phi_{V P}$, where $\phi_{H L}$ and $\phi_{V P}$ are the phases of the human leader and the VP, respectively. Note that positive values of $\phi$ correspond to the avatar following the human leader during the game. In addition, it is worth mentioning that velocity distributions were proposed recently as a signature of the human behavior during motor coordination [21].

We observe that, when using the reactive-predictive controller, the position of the virtual player presents oscillations away from the human participant position not only when he/she is moving, but also when he/she is still. Such an oscillatory feature does not appear when using the adaptive feedback controller. In general, both the position error $e=$ $x-r_{p}$ and the velocity error $\dot{e}=\dot{x}-\hat{r}_{v}$ turn out to be higher when using the RPC strategy. When using the adaptive feedback controller, the position error remains smaller never exceeding 0.2 , while it can become as high as 0.8 when using RPC. Similarly, the velocity error never exceeds 0.62 for the

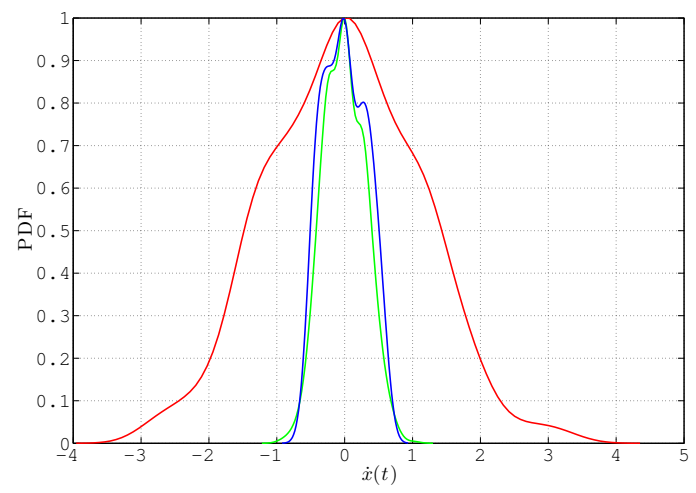

(a) veocity PDF

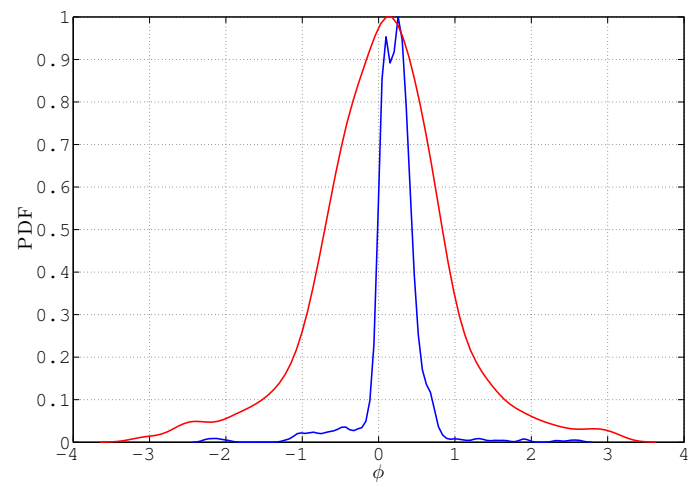

(b) relative phase PDF

Fig. 5. Distributions of the velocity (a) and the relative phase (b) between the human leader and the VP; blue (AFC), red (RPC), green (human leader)

AFC, while it goes up to a maximum of 3 for the RPC.

Moreover, when using AFC, the relative phase time series is much closer to 0 than that obtained when using RPC, meaning that with our proposed algorithm it is possible for the VP to better synchronize with the human leader. Such results are confirmed by the relative phase distributions obtained when using both the algorithms, as shown in Figure 5 (b). Finally, the difference in the velocity distributions of the human player and the VP is much more evident when RPC is used, confirming that our strategy better captures the features of the human player being able to replicate more accurately the kinematic properties observed in human motor coordination in the context of the mirror game.

\section{CONCLUSIONS}

After introducing the mirror game as a paradigm for the study of human motor coordination, we proposed a control algorithm to drive a virtual player able to play the game with a human leader. The control strategy is based on the use of a nonlinear HKB model and appropriate adaptive laws to determine the parameters of the coupling between the dynamics of the virtual player and the human sensed position and estimated velocity. Our results indicate that the adaptive feedback controller we propose performs well and guarantees better performance when compared to previous 
attempts in the literature, such as the reactive-predictive controller proposed in [1].

Specifically our approach is able to reduce both the position and velocity error between the human and the artificial player while also guaranteeing that the velocity distribution of the virtual follower is closer to that of the human leader.

We wish to emphasize that the derivation of control algorithms able to make artificial agents play the mirror game is a challenging problem in nonlinear control theory, although it is seldom studied. It is relevant in human-robot coordination and can be also extended to multi-player interactions (with or without the presence of humans).

A particularly challenging problem is that of making the dynamics of the virtual player resemble the movement of a human playing the game as much as possible. With this respect, we found that our current solution presents a reaction time which is shorter than the one shown by any human being. This might be overcome by adding appropriate delays in the control loop and is the subject of ongoing work to be presented elsewhere.

\section{ACKNOWLEDGMENT}

This work was funded by the European Project AlterEgo FP7 ICT 2.9 - Cognitive Sciences and Robotics, Grant Number 600610.

\section{REFERENCES}

[1] Noy, L., Dekel, E., Alon, U. (2011). The mirror game as a paradigm for studying the dynamics of two people improvising motion together. Proceedings of the National Academy of Sciences, 108(52), 2094720952.

[2] Zhang, J., Cheah, C. C., Collins, S. H. (2013). Stable human-robot interaction control for upper-limb rehabilitation robotics. In 2013 IEEE International Conference on Robotics and Automation (ICRA), (pp. 2201-2206).

[3] Folkes, V. S. (1982). Forming relationships and the matching hypothesis. Personality and Social Psychology Bulletin, 8(4), 631-636.

[4] Van Baaren, R. B., Holland, R. W., Kawakami, K., Van Knippenberg, A. (2004). Mimicry and prosocial behavior. Psychological science, 15(1), 71-74.

[5] Lakens, D., Stel, M. (2011). If they move in sync, they must feel in sync: Movement synchrony leads to attributions of rapport and entitativity. Social Cognition, 29(1), 1-14.

[6] Wiltermuth, S. S., Heath, C. (2009). Synchrony and cooperation. Psychological Science, 20(1), 1-5.

[7] Kelso, J. S., de Guzman, G. C., Reveley, C., Tognoli, E. (2009). Virtual partner interaction (VPI): exploring novel behaviors via coordination dynamics. PLoS One, 4(6), e5749.

[8] Dumas, G., de Guzman, G. C., Tognoli, E., Kelso, J. S. (2014). The human dynamic clamp as a paradigm for social interaction. Proceedings of the National Academy of Sciences, 111(35), E3726E3734.

[9] http://www.euromov.eu/alterego/.

[10] Bernstein, N. A. (1967). The co-ordination and regulation of movement. Oxford, Pergamon Press.

[11] Haken, H., Kelso, J. S., Bunz, H. (1985). A theoretical model of phase transitions in human hand movements. Biological cybernetics, 51(5), 347-356.

[12] Karst, G. M., Hasan, Z. (1991). Timing and magnitude of electromyographic activity for two-joint arm movements in different directions. Journal of Neurophysiology, 66(5), 1594-1604.

[13] Schöner, G., Haken, H., Kelso, J. A. S. (1986). A stochastic theory of phase transitions in human hand movement. Biological cybernetics, 53(4), 247-257.

[14] Diedrichsen, J., Shadmehr, R., Ivry, R. B. (2010). The coordination of movement: optimal feedback control and beyond. Trends in cognitive sciences, 14(1), 31-39.
[15] Jordan, M. I., Wolpert, D. M. (1999). Computational motor control. The Cognitive Neuroscience, Cambridge, MA: MIT Press.

[16] Kelso J. A. S., Scholz J. P. (1985). Cooperative phenomena in biological motion. In Complex Systems-Operational Approaches in Neurobiology, Physics, and Computers (pp. 124-149). Springer Berlin Heidelberg.

[17] Kay B. A., Kelso J. A. S., Saltzman E. L., Schöner G. (1987). Spacetime behavior of single and bimanual rhythmical movements: Data and limit cycle model. Journal of Experimental Psychology: Human Perception and Performance, 13(2), 178.

[18] Kelso J. A. S., Schöner G., Scholz J. P., Haken H. (1987). Phase-locked modes, phase transitions and component oscillators in biological motion. Physica Scripta, 35(1), 79.

[19] Jirsa, V. K., Fuchs, A., Kelso, J. A. S. (1998). Connecting cortical and behavioral dynamics: bimanual coordination. Neural Computation, 10(8), 2019-2045.

[20] Fuchs, A., Jirsa, V. K. (2008). JA Scott Kelso's contributions to our understanding of coordination. In Coordination: Neural, Behavioral and Social Dynamics (pp. 327-346). Springer Berlin Heidelberg.

[21] Hart, Y., Noy, L., Feniger-Schaal, R., Mayo, A. E., Alon, U. (2014) Individuality and togetherness in joint improvised motion, PLoS ONE, 9(2), e87213.

[22] Wang, W., Gao, Z. (2003). A comparison study of advanced state observer design techniques. In Proceedings of the 2003 American Control Conference, (Vol. 6, pp. 4754-4759).

[23] https://www.leapmotion.com/

[24] MATLAB R2012b (version 8.0). Natick, Massachusetts: The MathWorks Inc., 2012. 